\title{
Calculation of Excess Viscosities of Some Liquid Mixtures At 308 K Without Using The Theoritical Viscosity Values Of The Liquid Mixtures
}

\author{
Dikko A. B., Ahmed, A. D., Pascal T.
}

\begin{abstract}
The excess viscosity of liquids could only be calculated when the experimental and theoretical viscosity values were known. This paper explored the possibility of a method on how the excess viscosity of a liquid can be determined without the knowledge of its theoretical value. A capillary viscometer was used to get the experimental viscosities of distilled water, ethanol-water mixtures and methanol-water mixtures at $308 \mathrm{~K}$. The excess viscosities of some alcohol-water mixtures were then calculated using the modified Sunny Goh logarithmic viscosity equation.
\end{abstract}

\section{INTRODUCTION}

The term mixture is applied to a system in which the constituent particles are directly or indirectly distinguishable from one another. When the difference can be recognized directly or by the use of some optical magnifying device, it is customary to speak of mechanical mixtures, whilst the term solution is reserved for mixtures in which the constituent particles are of molecular dimensions, (Irving, 1977). There is no fundamental difference between the two classes of mixtures for they can all be resolved by the application of mechanical forces, for example, by a process of centrifuging. Gases are all completely miscible with one another, but miscibility in the liquid and solid states is much less in general. Some pairs of liquids are miscible in all proportions and complete series of mixed crystals are formed by certain pairs of crystalline solids. Other pairs of liquids and solid substances are practically immiscible. A third group is formed by pairs which are miscible in limited proportions. Miscibility depends upon the temperature, and in general is favoured by the chemical similarity of the substances concerned. Water does not mix with hydrocarbons, is miscible with methyl and ethyl alcohols, partially miscible with butyl and amyl alcohols, but practically immiscible with higher members of the alcohol series.

When two partially miscible liquid substances $\mathrm{A}$ and $\mathrm{B}$ are brought together, then, in general, two layers are formed which contain different proportions of A and B. The composition of these layers so-called conjugate pairs, (Wohlfarth, 2009) depends on the temperature. As a rule, the difference between the conjugates diminishes with rise of temperature and in some cases may ultimately disappear. The temperature at which this occurs is the critical solution temperature and above this the two substances are miscible in all proportions. Increased miscibility is sometimes produced by a fall of temperature and a few pairs of substances are known for which both upper and lower critical temperatures can be observed. Nicotine and water, for example, are completely miscible below $61^{\circ} \mathrm{C}$ and above $210^{\circ} \mathrm{C}$; between these limits they are only partially miscible.

Since the properties of pure liquids are largely dependent on the attractive forces between the molecules, it is extremely unlikely that the relations connecting the properties of liquid mixtures with those of their components can be of the same simple nature as those which characterize the corresponding gaseous systems. In some cases the mixing of two liquids is accompanied by very pronounced changes in volume, by a rise or fall of temperature and by other effects. For other liquid pairs, such effects are so small as to be scarcely measurable. The relations between the properties of liquid mixtures and those of their components may be conveniently shown by plotting the measured property against the composition of the mixture. The property-composition curves obtained in this way sometimes deviate but little from the straight line which corresponds with the simple mixture rule, but for other mixtures large deviations are found, and frequently the curves show a maximum or a minimum, (Hulya, 2000). These deviations result to the excess values which are the differences between the experimental and the theoretical values. Therefore, excess viscosity of any liquid could only be determined if the theoretical viscosity is known. However, the modification of the Sunny Goh logarithmic viscosity (Dikko,2014), makes it possible to calculate the excess viscosity of liquid without requiring it theoretical value.

\section{METHOD}

A capillary viscometer is an instrument used to measure the viscosity, or thickness, of a liquid by measuring how long it takes the liquid to flow through a small-diameter tube, or capillary. The flow, or efflux, time is directly proportional to the liquid's kinematic_viscosity. Viscosity is generally temperature dependent, so the capillary viscometer is usually used in a controlled-temperature water bath set to a specific temperature. 
Base on this fact, a capillary viscometer was locally constructed, (Dikko, 2014) and used to observe the time of flow of various alcohol-water mixtures between two marked points on the viscometer. Time $t$ was measured by a digital stop-watch with accuracy of $\pm 0.01 \mathrm{~s}$. The densities were determined by measuring the mass $(\mathrm{m})$ of a given volume $(\mathrm{v})$ of the mixture and using the relation, $\rho=\mathrm{m} / \mathrm{v}$. For determination of unknown water concentration in an alcohol sample we prepared different solutions containing different amounts of water in a fixed volume $(300 \mathrm{cc})$ of ethanol or methanol and the densities of all the liquid mixtures and pure liquids were experimentally determined.

Three readings for the time of flow, $\mathrm{t}_{2}$ were recorded for pure methanol, pure ethanol, and distilled water as well as for all the water-alcohol mixtures. The averages of the three readings were determined for different water concentrations, $\mathrm{C}_{\mathrm{w}}(\%)=\left(\mathrm{V}_{\mathrm{w}} / \mathrm{V}_{\mathrm{al}}\right) \times 100$. For distilled water the average of three such readings was recorded to be $t_{1}=1544.03 \pm 0.01$ seconds. The temperature during the experiments was recorded to be $308 \mathrm{~K}$. To determine the viscosity coefficient of a given liquid mixture using the equation (De and Dikko, 2012),

$$
\mu_{2}=\frac{\mu_{1} \rho_{2} t_{2}}{\rho_{1} t_{1}}=\mu_{\mathrm{ex}}
$$

we need the absolute value of $\mu_{1}$ of water at $308 \mathrm{~K}$. The values of $\mu_{1}$ at several temperatures are known (Dikko, 2014). By interpolating the value of $\mu_{1}$ at $308 \mathrm{~K}$ was found to be 7.22 millipoise. The density $\rho_{1}$ of water was taken to be $1 \mathrm{~kg} / \mathrm{m}^{3}$. The excess viscosity is calculated using the modified Sunny Goh logarithmic viscosity equation (Dikko 2014) as

$$
\ln (\mu)=\ln \left(\mu_{\infty}\right)+\frac{T_{e x}}{T} \ln \left(\frac{\mu_{e x}-\mu^{E}}{\mu_{\infty}}\right)
$$

and given in Tables (1 and 2). The plot of $\ln (\mu)$ against $1 / T$ will give a slope $=T_{\mathrm{ex}} \ln \left[\left(\mu_{e x}-\mu^{E}\right) / \mu_{\infty}\right]$ and intercept $=$ $\ln \left(\mu_{\infty}\right)$ on the $\ln (\mu)$ axis. It can be observed that the infinite viscosity can be determined from the intercept value $\ln \left(\mu_{\infty}\right)$ which is a constant, and the excess viscosity, $\mu^{E}$ can be determined from the slope of the plot of $\ln (\mu)$ versus $1 / T$ from equation $(2)$.

\section{RESULTS AND DISCUSSIONS}

The percentage water concentration in alcohol, experimental viscosity values, and the excess viscosity values determined are presented in tables 1 and 2

Table 1 Variation of Experimental and Excess Viscosity Coefficients of Ethanol-Water Mixtures with Water Concentration at $308 \mathrm{~K}$

\begin{tabular}{|c|c|c|}
\hline $\mathrm{C}_{\mathrm{w}}\left(\mathrm{v}_{\mathrm{w}} / \mathrm{v}_{\mathrm{a}}\right) 100 \%$ & $\begin{array}{c}\mu_{\mathrm{ex}} \\
\left(10^{-4} \mathrm{~Pa} . \mathrm{sec}\right) \pm 0.01\end{array}$ & $\begin{array}{c}\mu^{\mathrm{E}} \\
\left(10^{-4} \mathrm{~Pa} . \mathrm{sec}\right) \pm 0.01\end{array}$ \\
\hline 0 & 9.14 & 0.00 \\
\hline 5 & 9.25 & -0.23 \\
\hline 10 & 9.44 & -0.33 \\
\hline 15 & 9.60 & -0.43 \\
\hline 20 & 9.79 & -0.54 \\
\hline 25 & 9.95 & -0.57 \\
\hline 30 & 10.12 & -0.58 \\
\hline 35 & 10.27 & -0.59 \\
\hline 40 & 10.45 & -0.59 \\
\hline 45 & 10.61 & -0.57 \\
\hline 50 & 10.80 & -0.56 \\
\hline
\end{tabular}

$\mathrm{C}_{\mathrm{w}}=\mathrm{V}_{\mathrm{w}} / \mathrm{V}_{\mathrm{a}}(\%)=$ water concentration. $\mu_{\mathrm{ex}}=$ experimental viscosity coefficient. $\mu^{\mathrm{E}}=$ excess viscosity coefficient

Table 2 Variation of Experimental and Excess Viscosity Coefficients of Methanol-Water Mixtures with Water Concentration at $308 \mathrm{~K}$

\begin{tabular}{|c|c|c|}
\hline $\begin{array}{c}\mathrm{C}_{\mathrm{w}=} \\
\left(\mathrm{v}_{\mathrm{w}} / \mathrm{v}_{\mathrm{a}}\right) 100 \%\end{array}$ & $\begin{array}{c}\mu_{\mathrm{ex}} \\
\left(10^{4} \mathrm{~Pa} . \mathrm{sec}\right) \pm 0.01\end{array}$ & $\begin{array}{c}\mu^{\mathrm{E}} \\
\left(10^{-4} \mathrm{~Pa} . \mathrm{sec}\right) \pm 0.01\end{array}$ \\
\hline 0 & 4.91 & 0.00 \\
\hline 5 & 5.04 & -0.22 \\
\hline 10 & 5.13 & -0.37 \\
\hline 15 & 5.19 & -0.47 \\
\hline 20 & 5.30 & -0.53 \\
\hline 25 & 5.36 & -0.59 \\
\hline 30 & 5.47 & -0.61 \\
\hline 35 & 5.54 & -0.62 \\
\hline 40 & 5.64 & -0.62 \\
\hline 45 & 5.74 & -0.57 \\
\hline
\end{tabular}




\begin{tabular}{|l|l|l}
50 & 5.83 & -0.55 \\
\hline
\end{tabular}

$\mathrm{C}_{\mathrm{w}}=\mathrm{V}_{\mathrm{w}} / \mathrm{V}_{\mathrm{a}}(\%)$ = water concentration. $\mu_{\mathrm{ex}}=$ experimental viscosity coefficient. $\mu^{\mathrm{E}}=$ excess viscosity coefficient

All the systems show negative deviations of excess viscosities $\mu^{\mathrm{E}}$ from ideal over the entire concentration range. Minima correspond to $35-40 \%$ concentration, (Fig 1). As the concentration increases, the minima position also increases. As the molecular size decreases, the magnitude of the excess viscosity decreases, i.e., becomes more negative, showing a tendency to the ideal behaviour. It is interesting to note that for a situation of water being added to alcohol, it is the minima that correspond to $35-40 \%$ concentration. Generally, the curves of the excess viscosity versus water concentration in alcohol seem to be the opposite situation of alcohol concentration in water, (Hulya, 2000)

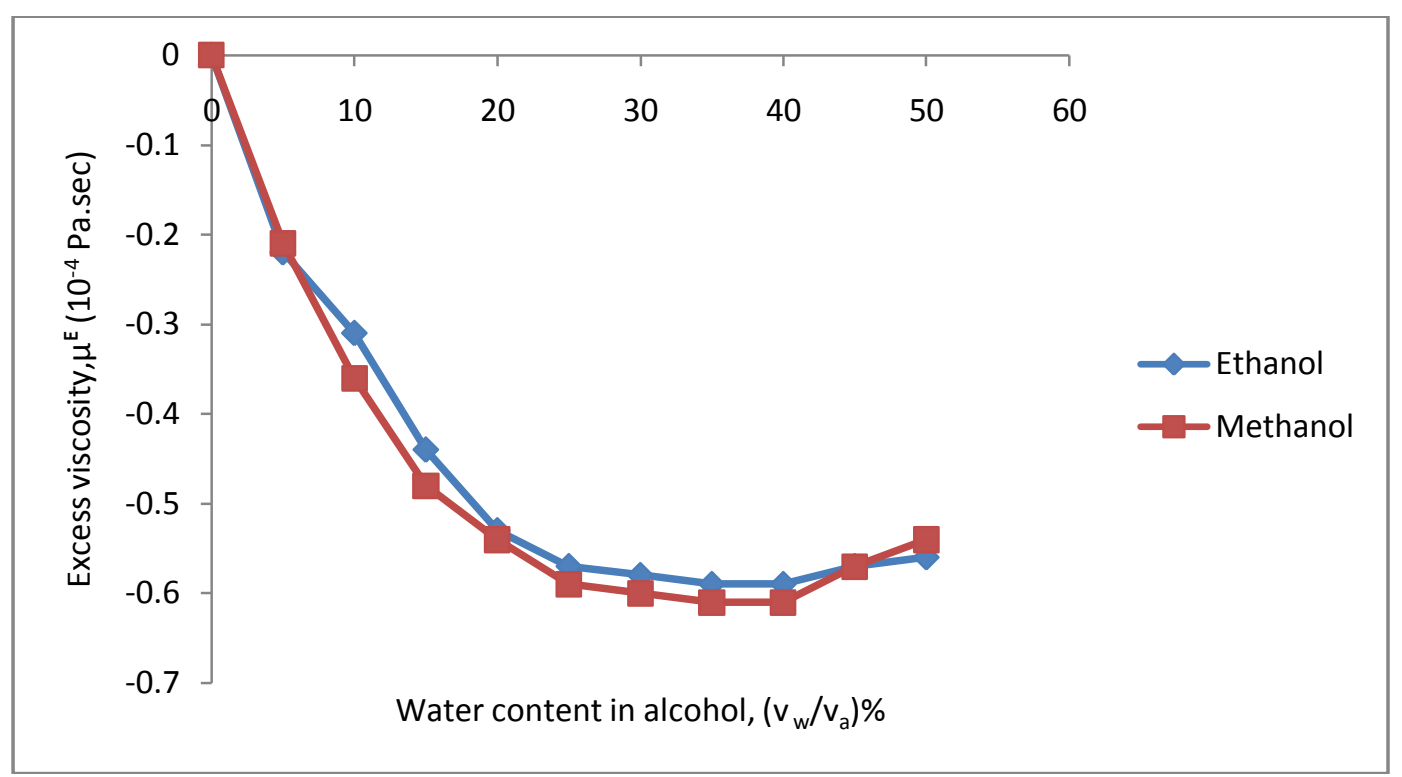

Fig 1 Variation of Excess Viscosity of Methanol and Ethanol with Water Content in Alcohol (Tables 1 and 2) at $308 K$

Various molecules may mix and dissolve in each other if they have approximately the same type of polarity. In the case of water and ethanol, this is the situation. The hydrogen of the -OH group on alcohol is polar as it is in the water molecule. Also, in solvents such as alcohol, which can take part in hydrogen bond formation, the self-association of alcohols may be increased in favour of hydrogen - bonded forms between solute and solvent.

The higher values of excess viscosities of alcohols in binary system may be due to the presence of larger alkyl chain. It seems that some kind of structural organization of water surrounding the hydrocarbon chain of alcohol is the most likely explanation of the observed dependence of viscosity on solvent composition, (Raza and Abbas, 2009, and Hulya, 2000). As can be understood from the above results, the excess properties of the studied mixtures indicate some molecular interactions. Because alcohols are associate liquids, they can interact with each other and with water molecules. The excess values of the viscosity exhibited a minimal which means a deviation from ideal behaviour. The negative values of the excess viscosity indicate that the alcohol molecules have greater influence in the flow than the water molecules in the mixture. Furthermore, the excess viscosity of any liquid mixture can now be calculated using the modified equation without requiring its theoretical value. The equation reveals that the infinite viscosity of a liquid or liquid-mixture is independent of temperature and concentration, while, the excess viscosity is dependent on both temperature and concentration.

\section{CONCLUSION}

If the excess viscosity values of any liquid or liquid-mixture is required, but its estimated viscosity value is not known, the modified equation has the solution. Just perform an experiment on any liquid and get the viscosity values of the liquid at several temperatures. Then get a plot of $\ln (\boldsymbol{\mu})$ versus $\mathbf{1} / \mathbf{T}$, (equation 2 ) and determine the infinite viscosity from the intercept, and in turn, excess viscosity from the slope. Thus, excess viscosity of any liquid can be determined without employing the theoretical viscosity value.

\section{REFERENCES}

[1] De D. K. and Dikko A. B., (2012), An innovative technique of liquid purity analysis and its application to analysis of water concentration in alcohol-water mixtures and studies on change of activation 
energies of the mixtures, Applied Physics Research, Canadian Center of Science and Education. Vol.4 No.4 pp. $98-114$

[2] Dikko A. B., (2014), Studies on the effects of solute concentration and temperature on certain physical properties of liquids for applications and analysis of liquid mixtures, PhD Thesis Presented to the Department of Physics, Modibbo Adama University of Technology, Yola.

[3] Hulya, Y. (2000), Excess Properties of Alcohol - Water Systems at 298.15 K. Turk J Phys 26 , pp 243 $=246$.

[4] Irving, J.B., (1977) "Viscosity of Binary Liquid Mixtures: The Effectiveness of Mixture Eq.s," National Engineering Laboratory Report, No. 630, East Kilbride, Glasgow, Scotland.

[5] Raza A. and Abbas M. (2009) Effect of Temperature and Solvent Composition on the Intrinsic Viscosity of Polyethyleneglycol in Water-Ethanol Mixture, American chemical society.

[6] Wohlfarth C. (2009), Viscosity of Pure Organic Liquids and Binary liquid Mixture. Lechner Manfred $\operatorname{Dieter}(E d)$, V II, pp 376 